\title{
Lactobacillus Murinus Alleviates Intestinal Ischemia/Reperfusion Injury Through Promoting the Release of Interleukin-10 From M2 Macrophages via Toll-like Receptor 2 Signaling
}

Jingjuan Hu

Southern Medical University Nanfang Hospital

Fan Deng

Southern Medical University Nanfang Hospital

Bingcheng Zhao

Southern Medical University Nanfang Hospital

Xiao Yang

Southern Medical University Nanfang Hospital

Zebin Lin

Southern Medical University Nanfang Hospital

Qishun Sun

Southern Medical University Nanfang Hospital

Mei Wu

Southern Medical University Nanfang Hospital

Shida Qiu

Southern Medical University Nanfang Hospital

Jin Zhao

Southern Medical University Nanfang Hospital

\section{Zhengzheng Yan}

Southern Medical University Nanfang Hospital

Weifeng Liu

Southern Medical University Nanfang Hospital

Cai Li

Southern Medical University Nanfang Hospital

Ke-Xuan Liu (D liukexuan705@163.com )

Southern Medical University Nanfang Hospital https://orcid.org/0000-0003-0221-366X

Research 
Keywords: intestinal ischemia/reperfusion injury, gut microbiota, lactobacillus murinus, macrophages, toll-like receptor 2, interleukin-10

Posted Date: February 8th, 2021

DOl: https://doi.org/10.21203/rs.3.rs-171684/v1

License: (c) (1) This work is licensed under a Creative Commons Attribution 4.0 International License. Read Full License

Version of Record: A version of this preprint was published at Microbiome on March 3rd, 2022. See the published version at https://doi.org/10.1186/s40168-022-01227-w. 


\section{Abstract}

\section{Background}

Intestinal ischemia/reperfusion (I/R) injury has high morbidity and mortality. Gut microbiota is one of the potential key factors affecting intestinal I/R injury. Different populations exhibit different sensitivities to intestinal I/R injury, but whether this is related to differences in gut microbiota is unclear. Here, to elucidate the interaction between the gut microbiome and intestinal I/R injury, we conducted 16S rRNA gene sequencing and fecal microbiota transplantation experiment in an I/R mouse model. Toll-like receptor 2 (TLR2)-deficient mice, interleukin-10 (IL-10)-deficient mice as well as the transwell co-culture system of small intestine organoids and macrophages were used to explore the potential mechanism of bacteria attenuating intestinal I/R injury.

Results

The intestinal I/R-sensitive (Sen) and resistant (Res) mice were first defined according to different survival outcomes of mice suffering from intestinal I/R. Fecal microbiota composition and diversity prior to intestinal ischemia were different between in Sen and Res mice. The relative abundance of Lactobacillus murinus (L. murinus) at the species level was higher in Res mice than in Sen mice, and the fold change was the largest. Clinically, the abundance of $L$. murinus in preoperative feces of patients undergoing cardiopulmonary bypass surgery was closely related to the degree of intestinal I/R injury after surgery. Treatment with $L$. murinus significantly prevented from intestinal I/R-induced intestinal injury and improved the survival of mice, which depends on the participation of macrophages. Further, in vivo and vitro experiments indicated that $L$. murinus promoted the release of IL-10 from M2 macrophages via activating TLR2 signaling to alleviate intestinal I/R injury.

\section{Conclusion}

Gut microbiome is involved in the postoperative outcome of intestinal I/R. L. murinus promotes the release of IL-10 from M2 macrophages through TLR2 signaling to alleviate intestinal I/R injury, revealing a novel mechanism of intestinal I/R injury and new therapeutic strategy for clinical practice.

\section{Background}

Intestinal ischemia reperfusion $(I / R)$ is a common but grave condition in some critical clinical settings such as acute mesenteric ischemia; hemorrhagic, or septic shock; severe burns; or some surgical procedures, including cardiopulmonary bypass (CPB), small bowel transplantation, and abdominal aortic surgery [1]. It not only causes local intestinal injury, but can also disrupt intestinal mucosal barrier [2], which allows enteric bacterial endotoxins to penetrate the blood and cause extraintestinal multiple organ dysfunction or even failure with high morbidity and mortality [3,4]. Currently, the mechanisms of intestinal I/R injury are not understood fully, and effective approaches for its clinical application are still lacking. 
The human gastrointestinal tract houses a vast and diverse intestinal microflora that provides nutrients and intrinsic immunity, regulates epithelial cell growth, and fundamentally affects human health and disease [5]. Few studies have reported the key role of the intestinal microbiome in intestinal I/R injury [6]. Our work stems from the previous observation that some SPF-free C57BL/ 6 mice survived 5 days after intestinal I/R, but some mice died quickly within $1 \mathrm{~h}$ after intestinal I/R. To better explore the above phenomenon, mice that died within 1 hour after reperfusion were defined as intestinal I/R-sensitive (Sen) mice, while those that survived up to 5 days were defined as intestinal I/R-resistant (Res) mice. These mice had the same genetic background, but their sensitivity to intestinal I/R injury was significantly different. We speculated that the different outcomes of mice suffering from intestinal I/R could be related to the difference in the gut microbiota. Furtherly, we conducted 16S DNA sequencing and fecal microbiota transplantation (FMT) experiment to study the intestinal bacterial community and specific bacterial strains in the intestinal I/R model of mouse. The results showed that the relative abundance of Lactobacillus murinus (L.murinus) at the species level was higher in the Res mice than in the Sen mice, suggesting that $L$.murinus could prevent from intestinal I/R injury. It has been demonstrated that $L$. murinus, as a potential probiotic, plays an important role in maintaining intestinal immune homeostasis in the mouse model of colitis through regulating T lymphocyte activity [7]. However, the role of L. murinus in intestinal $\mathrm{I} / \mathrm{R}$ injury remains unclear.

Toll-like receptor 2 (TLR2) and myeloid differentiation factor 88 (Myd88) are involved in the recognition of, and signaling in response to, a variety of pathogen-associated molecular patterns from gram-positive bacterium by innate immune cells including macrophages $[8,9]$. L.murinus is a gram-positive bacterium that can be used as a ligand for TLR2. It is unclear whether L.murinus plays a role in intestinal I/R injury through mediating TLR2 signaling on macrophages.

Interleukin-10 (IL-10) is an inflammatory and immunosuppressive factor and plays a vital role in controlling inflammation and preventing enteritis $[10,11]$. Its main sources are monocytes, macrophages and $T$ helper cells. Murine Kupffer cells are protective in total hepatic I/R injury with bowel congestion through the release of IL-10 [12]. However, the role of IL-10 secreted by macrophages during intestinal I/R injury is yet to be revealed.

Based on the above analysis, we hypothesized that gut microbiome is associated with the postoperative outcome of intestinal I/R, and L.murinus alleviates intestinal I/R injury through promoting the release of IL-10 from macrophages via activating TLR2 signaling. In the present study, we investigated the relationship between gut microbiome and postoperative outcome of intestinal I/R as well as the effects of L.murinus on intestinal I/R injury, and elucidated the potential mechanisms. This study will shed light on a novel mechanism of intestinal I/R injury related on gut microbiota and provide a potential therapeutic strategy in the clinical settings in the foreseeable future.

\section{Methods}

\section{Animal experiments}


All animal experimental procedures were carried out in accordance with the National Institutes of Health guidelines and were approved by the local Animal Care and Use Committee of the Nanfang hospital of Southern Medical University (Guangzhou, China). Six- to eight-week-old specific pathogen-free male C57BL/ 6 mice were purchased from the animal center of Nanfang Hospital of Southern Medical University (Guangzhou, China). TLR2 ${ }^{-/}$and IL-10 $10^{-/}$mice were purchased from Shanghai Model Organisms Center, Inc. All mice were housed under controlled temperature and humidity conditions with a 12-hour light-dark cycle, had free access to food and water and were fasted overnight before the experiment.

The model of intestinal I/R was established as described in our previous study [13]. Briefly, mice were anesthetized with $4 \%$ isoflurane, and a non-invasive microvascular artery clip was placed on the superior mesenteric artery (SMA) for 60 minutes (min) followed by clip removal for reperfusion of 120 min or 5 days. During the study period, body temperature was maintained at $37^{\circ} \mathrm{C}$ with a heating pad and liquid resuscitation was performed by injecting $0.5 \mathrm{ml}$ of physiological saline subcutaneously, just after reperfusion.

\section{Extraction and culture of organoids and the establishment of hypoxia-reoxygenation (H/R) models in vitro}

The extraction and culture of small intestinal organoids was performed as previously described $[14,15]$. The separated intestinal crypts were fixed onto the bottom of the dish with Matrigel (STEMCELL Technologies Inc., Shanghai, China) drops and covered with IntestiCult medium (STEMCELL Technologies Inc.). For the establishment of the organoid H/R model, the organoids were placed in a humid, anaerobic environment at $37^{\circ} \mathrm{C}$ for 12 hours and then placed in an aerobic environment containing $5 \% \mathrm{CO} 2$ in a $37^{\circ} \mathrm{C}$ incubator for 4 hours.

\section{Patient Samples}

From 2019 September to 2020 January, we recruited consecutive patients who underwent elective cardiac valve replacement or coronary artery bypass graft under cardiopulmonary bypass (CPB) at the Department of Cardiac Surgery, and healthy volunteers who underwent physical examination at the Department of Health Management, in Southern Medical University Nanfang Hospital, Guangzhou, China. Participants were not included if they (1) aged $<18$ or $>75$ years, (2) had chronic kidney disease, (3) had chronic digestive system diseases, previous gastrointestinal surgery, or confirmed or suspected intestinal ischemia/necrosis, (4) used antidiarrheals, laxatives or prebiotics within one week, or used antibiotics within 3 months. A total of 26 participants were enrolled, including 20 patients who underwent CPB and 6 healthy volunteers. There was no significant difference between the patient group and the healthy group in terms of demographic characteristics. The study protocol was approved by the Ethical Committee of Nanfang hospital, Southern Medical University (approval number NFEC-202009-k2-01).

Fecal and blood samples: Blood samples were collected at preoperatively (T0) and at $0 \mathrm{~h}$ (T1), $2 \mathrm{~h}$ (T2), 6 $\mathrm{h}$ (T3), $12 \mathrm{~h}$ (T4) and $24 \mathrm{~h}$ (T5) after surgery in EDTA plasma tubes as well as in serum separator tubes 
for analyses of intestinal fatty acid-binding protein (IFABP) and citrulline respectively. Fecal samples were collected at preoperatively, and the relative abundance of $L$. murinus was quantified by real-time PCR. IFABP and citrulline in the plasma samples were measured at multiple time points, to allow for of (T3-T0) concentration differences, respectively by means of a human IFABP ELISA Kit (Bio-Swamp, Wuhan, China) and Citrulline ELISA Kit (USCN, Wuhan, China), following the manufacturer's instructions. The gastrointestinal complication score of the patient on the seventh day after surgery was performed according to the acute gastrointestinal injury (AGI) standard described previously[16]. The detection of $L$. murinus, IFABP, citrulline and AGI scores were performed by researchers blinded to the group allocation.

\section{Microbe analysis}

Feces were collected with sterilized $1.5 \mathrm{ml}$ tubes before intestinal I/R and frozen at $-80^{\circ} \mathrm{C}$ until DNA extraction. All extracted DNA was stored in $-20^{\circ} \mathrm{C}$ until further test. The extracted fecal DNA concentration was diluted to $10 \mathrm{ng} / \mu \mathrm{l}$ and quantitative real-time polymerase chain reaction (PCR) was processed according to 16S rRNA primers, Firmicutes primers and Bacteroidetes primers, the primers were listed in Table S1. Moreover, 16S rRNA abundance from blood was normalized to host $18 \mathrm{~S}$.

\section{S rRNA gene sequencing}

Microbial DNA from preoperative fecal samples of mice were extracted using the classical phenolchloroform extraction method as previously described $[17,18]$. Next, the V4 region of $16 \mathrm{~S}$ rDNA was amplified using barcode specific primers (V4F, 5'-GTGTGYCAGCMGCCGCG GTAA-3' and V4R, 5'CCGGACTACNVGGG TWTCTAAT-3'), and the amplified products were quantified using QuantiFluorTM and mixed in equal amounts.

All samples were paired-end sequenced on the Illumina Hiseq PE2500 sequencing platform. Low-quality reads were filtered after quality control, and high-quality reads were assigned to operational taxonomic units (OTUs) of $\geq 97 \%$ similarity using UPARSE pipeline [19]. QIIME was applied to analyze the alpha and beta diversities, based onweighted and unweighted UniFrac distances successively Metastasis (version 20090414) and Linear discriminant effect size (LEfSe) software[20] (version 1.0) were used to explore biomarker features in each group. The KEGG pathway analysis of the OTUs was performed using Tax4Fun[21] (version 1.0) and was performed using the OmicShare tools, a free online platform for data analysis (www.omicshare.com/tools). The calculated $p$-value was gone through FDR Correction, taking FDR $\leq 0.05$ as a threshold.

\section{Bacterial Strains and Growth Conditions}

L. murinus freeze-dried powder (BN, Beijing, China) was dissolved with $0.5 \mathrm{ml}$ MRS medium (HKM, Guangdong, China), then the bacteria liquid was coated on the blood plate, and bacterial colonies appeared after about $24 \mathrm{~h}$. Single colonies were picked into MRS medium, were incubated at $37^{\circ} \mathrm{C}$ under anaerobic conditions, and $O D 600=0.6-0.7$ of cultures was measured until mid-log phase after $12-16 \mathrm{~h}$ of growth, at which time the colony count was $6.8 \times 10^{8} \mathrm{CFU} / \mathrm{mL}$ by plate count. Frozen stocks of $L$. murinus 
(in MRS medium with $25 \%$ glycerol) were prepared, stored at $-80^{\circ} \mathrm{C}$ for further experiments. $50 \mu$ frozen stocks of L. murinus were added to $5 \mathrm{ml}$ MRS medium and incubated at $37^{\circ} \mathrm{C}$ under aerobic conditions for $12 \mathrm{~h}$, and then used for gavage of mice. In order to evaluate the total amount of L. murinus DNA in cecum and stool samples, was quantified by quantitative real-time PCR using the following primers: $L$. murinus[22], LactoM-F (5'-TCGAACGAAACTTCTTTATCACC-3') and LactoM-R (5'CGTTCGCCACTCAACTCTTT-3').

\section{Experimental design}

L. murinus pretreatment experiment: As shown in Additional file 1a, mice were randomly divided into 3 groups: (1) Sham group; (2) I/R group; (3) I/R+L. murinus group. The sham group of mice were gavaged daily for 7 days with $200 \mu$ MRS medium and then intestinal I/R was performed without SMA occlusion. The I/R group of mice were gavaged daily for 7 days with $200 \mu$ I MRS medium, and intestinal SMA was occluded for $60 \mathrm{~min}$ followed by $120 \mathrm{~min}$ reperfusion. The l/R+ L. murinus group of mice were gavaged daily for 7 days with $200 \mu \mathrm{l} 6.8 \times 10^{8} \mathrm{CFU} / \mathrm{ml}$ L. murinus prior to establishing intestinal I/R. In addition, to explore a single strain of $L$. murinus alleviates intestinal I/R-induced intestinal injury, mice were allocated randomly to 2 groups: Group $A B X+I / R$, in which antibiotics $(A B X)$ was administered $1 \mathrm{w}$ before intestinal ischemia; Group $A B X+1 / R+L$. murinus, treatment of mice with $A B X$ for a week, and then mice were gavaged daily for 7 days with $200 \mu \mathrm{l} 6.8 \times 10^{8} \mathrm{CFU} / \mathrm{ml}$ L. murinus prior to establishing intestinal I/R.

Macrophage depletion experiment: Mice were allocated randomly to 4 groups: (1) I/R group; (2) PBS+Lipo+I/R group; (3) Clodronate-Lipo+I/R group; (4) Clodronate-Lipo+L. murinus+I/R group. I/R group of mice, in which intestinal SMA was occluded for 60 min followed by 120 min reperfusion was established as described previously; PBS+Lipo+l/R group of mice, which were intraperitoneally injected with $200 \mu$ l empty liposomes (Yeasen, Shanghai, China) $24 \mathrm{~h}$ before establishing I/R; Clodronate-Lipo+I/R group of mice, which were intraperitoneally injected with $200 \mu$ l clodronate liposomal (Yeasen, Shanghai, China) $24 \mathrm{~h}$ before establishing I/R model; Clodronate-Lipo+L. murinus+l/R group of mice, which were given treatment of $L$. murinus and clodronate liposomal injection before establishing I/R (Additional file 1b).

To explore L. murinus alleviates intestinal injury through TLR2 on the surface of macrophages, wild type (WT) mice were randomly divided into the following groups (Additional file 1c): (1) Sham; (2)I/R; (3)I/R+L. murinus. TLR2 ${ }^{-/}$mice were randomly divided into the following groups: (1)l/R; (2)I/R+L. murinus.

To explore L. murinus promotes the release of IL-10 from macrophages through TLR2 to alleviate intestinal I/R injury, wild-type (WT) mice were randomly divided into the following groups (Additional file 1d): (1)I/R; (2)I/R+L. murinus. IL-10-- mice were randomly divided into the following groups: (1)I/R; (2) $I / R+L$. murinus.

To explore $L$. murinus promotes the release of IL-10 by macrophages through TLR2 to reduce organoid $\mathrm{H} / \mathrm{R}$ injury, we established the transwell co-culture system of macrophages separately extracted from WT, 
or $\mathrm{TLR}^{-/-}$or IL-10 ${ }^{-/-}$mice and small intestinal organoids extracted from WT mice. In our experiment, the transwell chamber $(0.4 \mu \mathrm{m}$ pore size polyester membrane; Corning, Inc.) were placed in a 6-well culture plate, small intestinal organoids were planted in the upper chamber, and macrophages were planted in the lower chamber. The $200 \mu$ supernatant of $L$. murinus stimulates the upper chamber to further confirm the $L$. murinus may require the participation of macrophages to improve the intestinal injury in vitro.

\section{Fecal microbiota transplantation}

Fecal microbiota transplantation (FMT) was performed according to the modified method described previously [18]. Briefly, 6-8-week-old male C57BL/6 mice were given antibiotics (vancomycin, $100 \mathrm{mg} / \mathrm{kg}$; neomycin sulfate $200 \mathrm{mg} / \mathrm{kg}$; metronidazole $200 \mathrm{mg} / \mathrm{kg}$; and ampicillin $200 \mathrm{mg} / \mathrm{kg}$ ) intragastrically once each day for 1 week to deplete the gut microbiota (receptor mice). Preoperatively collected feces of sensitive and resistant mice or low $L$. murinus patient feces and high $L$. murinus patient feces (donor mice) were resuspended in PBS at $0.125 \mathrm{~g} / \mathrm{ml}$. An amount of $0.1 \mathrm{ml}$ of the solution was administered to mice in the corresponding groups orally via gastric gavage tube 1 week. All the mice had free access to food and water, and mice were performed intestinal ischemia $1 \mathrm{~h}$ and reperfusion $2 \mathrm{~h}$ surgery after 1 weeks of transplantation, and then blood, ileum, kidney, lung and liver samples were harvested in a sterile manner for further examination.

\section{Gene expression analysis}

A reverse transcript enzyme (TOYOBO, Tokyo, Japan) was applied to prepare CDNA according to the manufacturer's protocol. The real-time PCR reaction was performed using the ABI Q5 real-Time PCR System with SYBR Green detection protocol (TOYOBO, Tokyo Japan). The expression of target genes in mice were normalized to house-keeping gene $18 \mathrm{~S}$ using the $2^{-\wedge \wedge} \mathrm{CT}$ method. The target genes primers were listed in Additional file 2

\section{Protein expression and biochemical analysis}

The endotoxin level was measured via commercial kit (GenScript, Nanjing, China). Plasma AST was determined manually with a commercial kit (KeyGene, Nanjing, China). The protein level of IL-10 in the ileum was measured by IL-10 ELISA kit (ABdonal, Wuhan,China).

\section{Histological staining}

lleum, liver, lung and kidney samples tissue were collected and fixed in $4 \%$ paraformaldehyde. Then, the samples were embedded in paraffin, 5 - $\mu$ m-thick sections were sliced and were stained with hematoxylineosin (HE) according to the experimental protocol. The degree of ileum injury after reperfusion was evaluated using a modified Chiu method [23] according to changes of the intestinal mucosa villus and glands. Liver, lung and kidney tissue histological damage were assessed according to previously described scoring system $[24,25,26]$. Images were captured at $200 \mathrm{X}$ with Olympus fluorescence microscope. (Olympus, Japan). 


\section{Immunofluorescence}

Paraffin section samples were generated as above, blocked for $1 \mathrm{~h}$, and incubated overnight at $4{ }^{\circ} \mathrm{C}$ with anti-ZO-1 antibody (Abcam, Cambridge, MA, USA) and anti-Occludin antibody (Abcam, Cambridge, MA, USA). Tissues were then washed, stained with DAPI for $10 \mathrm{~min}$, and Images were captured at $200 \mathrm{X}$ with fluorescent microscopy (Olympus, Japan). Quantification of the fluorescence intensity of ZO-1 and Occludin staining were performed by automated image analysis in five randomly chosen $200 \mathrm{X}$ fields of each sample.

\section{Peritoneal Macrophage Collection}

Injection of $4 \mathrm{ml}$ of normal saline solution into the peritoneum was used for peritoneal macrophage collection, and the mice's abdomen was gently rubbed for $2 \mathrm{~min}$ to make the liquid flow in the abdominal cavity. The peritoneal fluid was sucked out and transferred into a centrifugal tube with a glue-head dropper. The amount of each suction was about $4 \sim 5 \mathrm{ml}$. The collected peritoneal lavage fluid was centrifuged at $1000 \mathrm{r} / \mathrm{min}$ for $10 \mathrm{~min}$ and the supernatant was removed, then further flow experiment was carried out.

\section{Flow Cytometry and Antibodies}

Peritoneal macrophages were washed and stained with $0.25 \mu \mathrm{g}$ of anti-mouse F4/80 Antigen FITC (Invitrogen eBioscience, USA), PE-Cy7 anti-mouse CD45 (BD Bioscience, USA), APC anti-mouse CD206 (Invitrogen eBioscience, USA), anti-mouse CD11C (Invitrogen eBioscience, USA), BV421 anti-mouse CD282هTLR2 (BD Bioscience, USA) and BV510 anti-mouse IL-10 (BD Bioscience, USA). F4/80 ${ }^{+}$CD $45^{+}$ served as a macrophage marker, with $\mathrm{F} 4 / 80^{+} \mathrm{CD} 45^{+} \mathrm{CD} 206^{+} \mathrm{CD} 11 \mathrm{C}^{-}$cells considered to be $\mathrm{M} 2$ macrophages and $\mathrm{F} 4 / 80^{+} \mathrm{CD} 45^{+} \mathrm{CD} 206^{-} \mathrm{CD} 11 \mathrm{C}^{+}$cells considered to be $\mathrm{M} 1$ macrophages. Flow cytometric analysis was performed on FACS Calibur (BD Biosciences, USA) instruments and analyzed using FlowJo software (Tree Star Inc.).

\section{Detection of organoid injury by lactate dehydrogenase (LDH) assays}

The LDH kit (Nanjing Jiancheng Bioengineering Institute, Nanjing, China) was used to detect the level of LDH in the culture medium to assess organoid damage. The detection of LDH was carried out based on the manufacturers' protocols.

\section{Statistical analysis}

All analyses were performed using GraphPad Prism software (version 7.0). Data are presented as means \pm standard error of mean (SEM). Statistical analyses were performed using two-sided Student's $t$ tests or by one-way analysis of variance (ANOVA) as indicated in the figure legends. $p$ values $<0.05$ were considered statistically significant.

\section{Results}




\section{Characteristics of tissue injury and the gut microbiota in intestinal Sen and Res mice.}

Feces from C57BL/6 mice were collected before intestinal I/R following which the mice underwent intestinal ischemia and the survival rate was observed after reperfusion. Mice that died within $1 \mathrm{~h}$ after reperfusion were defined as Sen mice, while those that survived up to 5 days were defined as Res mice (Fig. 1a). Compared with the Res mice, the Sen mice showed significantly aggravated HE injury and scores in the ileum, liver, lung and kidney as well as up-regulated mRNA levels of $I L-1 \beta, I L-6$ and TNF- $a$. (Fig. 1b, c and Additional file 3a). Moreover, the mRNA levels of intestinal tight junction markers in the ileum of the Res mice were markedly higher, whereas, the plasma endotoxin level was reduced compared with those in Sen mice (Fig. 1d, e).

Real-time PCR and 16S DNA sequencing were used to explore whether the susceptibility or resistance of the Sen or Res mice, respectively, to intestinal I/R was related to differences in their gut microflora. There was a significant increase in the relative total bacterial load; the relative abundance of Firmicutes and Bacteroidetes as well as the Firmicutes/Bacteroidetes ratio in the feces of Sen mice were lower than those in the feces of Res mice (Additional file 3b, c). 16S DNA sequencing results showed that the bacterial composition in fecal samples from Sen and Res mice in terms of both bacterial phyla and class were significantly different (Fig. $1 \mathrm{f}$ and Additional file $3 \mathrm{~d}$ ). Alpha diversity analysis and principal coordinates analysis (PCoA) indicated that the overall structure of the gut microbiota was significantly different between the Sen and Res mice (Fig. 1g, h). To further explore the differences of gut microbial metabolic function in Sen and Res group, the 16S rRNA data were annotated with metabolic pathways from the KEGG data using Tax4Fun prediction analysis. The relative abundances of 20 metabolism related KEGG pathways were shown in Pathway abundance heat map (Additional file 3e). In particularly, the relative KEGG pathways abundances of "Metabolism; Energy Metabolism; Oxidative phosphorylation(ko00190)" and "Environmental Information Processing; Membrane Transport; Bacterial secretion system(ko03070) were statistically upregulated in the Sen group compared with those of the Res group. LEfSe analysis indicated that the higher relative abundance of Coprostanolignes-group and Ruminococcaceae-UCG-010 at the genus level in the Res mice than in the Sen mice was statistically significant (linear discriminant analysis [LDA] score $>2$, Fig. 1i). Moreover, at the species level, the relative abundance of $L$. murinus in the Res mice was significantly higher than in the Sen mice, and the fold change was the largest (Fig. $1 \mathrm{j}$ ). The relative abundance of L. murinus was negatively correlated with inflammatory factors of intestinal I/R injury (Fig. 1k).

\section{Gut microbiota from Res mice could independently alleviate I/R-induced tissue damage}

To further demonstrate that the gut microbiota plays an important role in intestinal I/R injury, FMT experiment was performed (Fig. 2a). Recipient mouse feces were collected on the third and seventh days after FMT. The mice that received feces from Sen mice (Sen feces group) showed marked decrease in the levels of Firmicutes and increase in the relative total bacterial load compared with the mice that received feces from Res mice (Res feces group) (Fig. 2b, c). The above results demonstrated that the FMT experiment was successful. 
Intestinal I/R (60 min/120 min) were performed, after a week of gavage. The Res feces group had significantly reduced intestinal I/R-induced HE injury and decreased mRNA levels of $I L-1 \beta, T N F-a, C X C l 2$, $C c / 2$ and $C c / 5$ in the ileum compared with the Sen feces group (Fig. 2d, e). As presented in Fig. $2 f-h$, the Res feces group had markedly up-regulated mRNA and protein levels of ZO-1 and Occludin in the ileum and reduced plasma endotoxin level compared with the Sen feces group. Further, the Res feces group had significantly reduced HE injury and down-regulated mRNA levels of inflammatory factors in the liver, lung and the kidney when compared with the Sen feces group (Fig. 2i-I). The FMT experiment confirmed that the gut microbiome prior to intestinal ischemia is closely related to the postoperative outcome of intestinal I/R.

\section{A single strain of $L$. murinus alleviates intestinal $\mathrm{l} / \mathrm{R}$-induced intestinal injury}

From the 16S rRNA gene sequencing analysis, it was found that the relative abundance of $L$. murinus was four times higher in the Res mice than in the Sen mice (Fig. 1j). Moreover, the relative abundance of this species decreased significantly after intestinal I/R, as evidenced by from real-time PCR (Fig. 3a). This prompted us to investigate whether oral administration of $L$. murinus could ameliorate intestinal I/Rinduced intestinal injury. Treatment with L.murinus significantly increased the relative abundance of $L$. murinus in the cecum, improved survival rate, and reduced HE injury compared with those in I/R mice (Fig. 3a-c). The l/R + L.murinus and sham mice had markedly increased ZO-1 and Occludin mRNA and protein levels compared with I/R mice (Fig. $3 \mathrm{~d}-\mathrm{g}$ ). To further demonstrate the role of a single strain of $L$. murinus in intestinal I/R, mice were treated with $A B X$ and then were gavaged for 7 days with $L$. murinus before I/R (Supplementary Fig. 1a). Real time PCR revealed that the $A B X+L$. murinus $+1 / R$ group had significantly up-regulated relative abundance of $L$. murinus compared with the $A B X+1 / R$ group (Fig. $3 \mathrm{~h}$ ). As presented in Fig. 3i-I, L. murinus reduced HE injury, while increasing ZO-1 and Occludin mRNA and protein levels in the ileum following I/R. The above results demonstrated that $L$. murinus alleviates intestinal I/R-induced intestinal injury in mice.

\section{Relationship between $L$. murinus and postoperative intestinal injury in patients undergoing cardiopulmonary bypass surgery.}

In patients undergoing cardiac surgery, CPB is potentially responsible for the reduced intestinal ischemia (reduced blood supply and oxygen delivery) and injury; thus, it was used as a clinical model of intestinal $\mathrm{I} / \mathrm{R}$ in this study $[27,28]$. Firstly, the relative abundance of $L$. murinus in preoperative feces of patients undergoing CPB surgery was less than 0.1 and more than 0.1 , which were categorized into low and high L. murinus abundance, respectively (Fig. $4 a, b$ ). There were no differences in baseline characteristics between the two groups (Additional file 4). Plasma concentrations of citrulline and intestinal fatty acidbinding protein IFABP in both groups were measured as markers of absorptive enterocyte mass and intestinal failure in humans $[29,30]$. There were no significant differences in the levels of citrulline and IFABP between the two groups before surgery (Fig. 4c). Surprisingly, as shown in Fig. 4d-f, the abundance of $L$. murinus in preoperative feces of patients undergoing CPB surgery was negatively correlated with changes in IFABP concentration and positively correlated with changes in citrulline concentration at 6 
hours after operation. The gastrointestinal function of the patients was further evaluated after 7 days post operation, and the occurrence rate of gastrointestinal injury in the low L. murinus abundance group patients was $60 \%$, while the high L. murinus abundance group patients remained clinically devoid of gastrointestinal complications (Fig. 4g).

To further prove the role of $L$. murinus in ameliorating intestinal injury, FMT experiment was carried out where fecal bacteria from the high and low L. murinus abundance groups of patients collected preoperatively were transplanted into mice separately and were categorized correspondingly into high $L$. murinus feces group and low $L$. murinus feces group, respectively. Real-time PCR revealed that the relative abundance of $L$. murinus in feces of the high $L$. murinus feces group was markedly higher than that in the low L. murinus feces group after FMT (Fig. 4 h). HE staining revealed that the high $L$. murinus feces group had markedly reduced I/R-induced intestinal damage, but increased mRNA and protein levels of ZO-1 and Occludin in the ileum compared with the low L. murinus feces group (Fig. 4i-k).

\section{Improvement in intestinal I/R injury by L. murinus depends on macrophage participation}

The above animal and clinical experimental results have verified that $L$. murinus has a protective effect on in intestinal I/R injury, but the mechanism has not yet been elucidated. L. murinus affects immune response, but whether it regulates macrophages in the intestinal I/R is unclear. Flow cytometry results showed that intestinal I/R induced a significant increase in the number of macrophages $\left(F 4 / 80^{+} \mathrm{CD} 45^{+}\right)$, which was reversed by $L$. murinus treatment (Fig. 5a). To further verify whether the protective effect of $L$. murinus is related to macrophages, clodronate liposomal was administered intraperitoneally to mice two days before I/ $R$ to deplete macrophages (Supplementary Fig. 1b). The Clodronate-Lipo $+\mathrm{I} / \mathrm{R}$ group exhibited decreased the number of macrophages (Fig. 5b) and reduced I/R-induced HE injury (Fig. 5c, d), as well as up-regulated mRNA and protein levels of ZO-1 and Occludin (Fig. 5c, e-f) compared with the I/R mice and the PBS + Lipo + l/R group, but showed no statistical difference when compared with the Clodronate-Lipo + L. murinus + I/R group. Hypoxia-reoxygenation (H/R) model of small intestinal organoids was established to further confirm the results of in vivo experiments. As shown in the Fig. $5 \mathrm{~g}$, $h, L$. murinus reduced morphological damage of intestinal organoids injury and LDH levels, but increased the mRNA levels of ZO-1 and Occludin (Fig. $5 \mathrm{i}$ ) in the co-culture system of macrophages and organoids following $\mathrm{H} / \mathrm{R}$, but had no obvious effect on these data in the organoids cultured alone. Consistent with the results of in vivo experiments, the protective effect of $L$. murinus on intestinal I/R injury may depend on the participation of macrophages.

\section{L. murinus promotes the release of IL-10 from M2 macrophages through activating TLR2 signaling to alleviate intestinal $\mathrm{l} / \mathrm{R}$ injury}

As shown in the Fig. 6a, flow cytometry results showed that intestinal I/R induced a significantly decreased the proportion of M2 macrophages (CD11C ${ }^{-}$CD206 $\%$ ) in peritoneal, which was reversed by $L$. murinus treatment in WT mice. In addition, intestinal I/R induced a significant increase in the expression of TLR2 on the surface of M2 macrophages, which was enhanced by L. murinus treatment. TLR2 ${ }^{-/-}$mice 
was used to explore the effect of TLR2 on the surface of M2 macrophages in the protection of L. murinus on intestinal I/R injury. L. murinus significantly increased the proportion of M2 macrophages and TLR2 ${ }^{+}$ M2 macrophages/total M2 macrophages in WT mice following intestinal I/R, but not in TLR2 ${ }^{-/-}$mice (Fig. 6a). Compared with in the I/R group, L. murinus significantly reduced HE injury and scores in WT mice, but in $\mathrm{TLR}^{-/-}$mice, the protective effect of $L$. murinus on intestinal I/R injury disappeared (Fig. 6b). Consistent with the changes in TLR2 expression on the surface of macrophages, the mRNA levels ZO-1 and Occludin (Fig. 6c, d) in the ileum of mice in the $1 / R+L$. murinus group were higher than those in the ileum of mice in the I/R group in WT mice, but not in TLR2 ${ }^{-/-}$mice. Furthermore, the proportion of IL-10 M2 macrophages/total M2 macrophages in peritoneal, the mRNA and protein levels of IL-10 in the ileum of mice in the $\mathrm{I} / \mathrm{R}+L$. murinus group were higher than those of mice in the $\mathrm{I} / \mathrm{R}$ group in WT mice, but not in TLR2 ${ }^{-/-}$mice (Fig. 6e-g). These indicated that L. murinus reduced intestinal I/R injury through TLR2, accompanied by an increase in the proportion of IL-10+ $\mathrm{M} 2$ macrophages.

Then IL-10 $10^{-/}$mice were used to explore the role of IL-10 in the protection offered by $L$. murinus in intestinal I/R injury. We found that the mRNA and protein levels of IL-10 in the ileum of the I/R $+L$. murinus group were higher than those in the I/R group in WT mice, while the mRNA and protein levels of $\mathrm{IL}-10$ in IL-10/- mice were drastically reduced (Fig. 6h, i). Compared to the I/R group, L. murinus treatment and rmIL-10 treatment significantly reduced $\mathrm{HE}$ injury and scores, as well as increased the mRNA levels of ZO-1 and Occludin in WT mice. However, L. murinus treatment had no obvious effect on HE injury and scores, the mRNA levels of ZO-1 and Occludin in IL-10-/- mice following intestinal I/R (Fig. $6 \mathrm{j}, \mathrm{k}$ ). The above results indicated that $L$. murinus promotes the release of IL-10 from macrophages through TLR2 to alleviate intestinal I/R injury in mice.

\section{L. murinus alleviates organoid $H / R$ injury through promoting the release of IL-10 from macrophages via} TLR2 signaling.

As we showed that the protective effect of $L$. murinus on organoid injury depends on the participation of macrophages (Fig. $5 \mathrm{~g}-\mathrm{i}$ ), we decided to explore the specific mechanism of macrophages in small intestinal organoids. A transwell co-culture system of macrophages extracted from TLR2 ${ }^{-/-}$mice (TLR2 ${ }^{-/}$

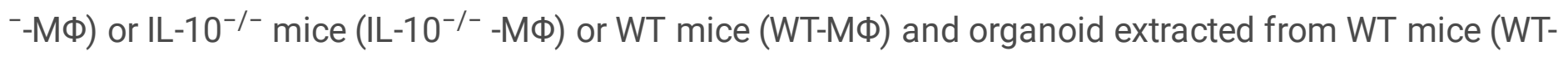
Org) was established. It was uncovered that $L$. murinus treatment increased the proportion of M2 macrophage, the expression of TLR2 on the surface of M2 macrophages and the mRNA level of Myd88 compared with H/R group in the co-culture system of WT-MФ and WT-Org, but not in the co-culture system of TLR2 ${ }^{-/-}-M \Phi$ and WT-Org (Fig. 7a-c). L. murinus reduced organoids HE injury and LDH levels, while increased the mRNA and protein levels of ZO-1 and Occludin in the co-culture system of WT-MФ and WTOrg, but not in the co-culture system of TLR2 ${ }^{-/-}-\mathrm{M} \Phi$ and WT-Org (Fig. 7d-g).In addition, flow cytometry results showed that $L$. murinus suppressed the decrease in the proportion of IL-10+ $\mathrm{M} 2$ macrophages/total M2 macrophages induced by $\mathrm{H} / \mathrm{R}$ in the transwell co-culture system of WT-M $\Phi$ and WT-Org, but not in the transwell co-culture system of TLR2 ${ }^{-/-}-\mathrm{M} \Phi$ and WT-Org, IL-10-/- $-\mathrm{M \Phi}$ and WT-Org (Fig. 7h). The co-culture system of IL10 $10^{-/-}-\mathrm{M} \Phi$ and WT-Org was established to explore the role of IL-10 
released by macrophages in the protective effect of $L$. murinus on organoid $\mathrm{H} / \mathrm{R}$ injury. L. murinus reduced organoids HE injury and LDH levels, while increased the mRNA and protein levels of ZO-1 and

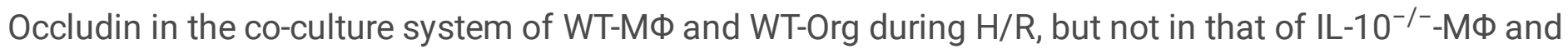
WT-Org (Fig. 7i-I). The above results indicated that $L$. murinus promotes the release of IL-10 from M2 macrophages through TLR2 to alleviate organoid H/R injury in the co-culture system of macrophages and organoids.

\section{Discussion}

In the present study, we for the first time confirmed that mice with the same genetic background had huge differences in sensitivity to intestinal I/R injury, which, at least partly, results from the difference in the gut microbiome in the feces of mice before intestinal ischemia. Furtherly, our results revealed that the relative abundance of $L$. murinus at the species level in the feces from the Res mice before intestinal ischemia was significantly higher than that in the Sen mice, and was negatively correlated with the levels of inflammatory factors following intestinal I/R. Clinical evidence also showed that the abundance of $L$. murinus in preoperative feces of patients undergoing CPB surgery was closely related to the degree of intestinal I/R injury after surgery. FMT indicated that the abundance of $L$. murinus in preoperative feces of patients is related to different sensitivities of mice to intestinal I/R injury. More importantly, treatment with $L$. murinus significantly prevented from intestinal I/R-induced intestinal injury and improve the survival of mice, which depends on the participation of macrophages. Another important finding of the current study was that $L$. murinus promotes the release of IL-10 from M2 macrophages through activating TLR2 signaling to alleviate intestinal I/R injury, which shed light on a novel mechanism of intestinal I/R injury and new therapeutic strategy for clinical practice.

It is well known that patients suffering from shock or undergoing some surgical procedures including CPB surgery have different outcome after receiving medical treatment. The reason for the difference has remained being unclear. In the present study, we employed the mouse model of intestinal I/R and identified the difference that mice with the same genetic background have significant differences in sensitivity to intestinal I/R injury. Especially, 16S DNA sequencing and FMT results showed that gut microbiome is involved in postoperative outcome of intestinal I/R. This is a novel finding.

Gut microbiota is a complex ecosystem susceptible to the surrounding environment and diet. At present, only a few studies have reported the changes of gut microbiota in intestinal I/R by the traditional DGGE method [31]. Previous antibiotic experiments have shown that depletion of gut commensal bacteria can attenuate intestinal I/R injury [32]. However, the extensive diversity of the gut microbiome makes it difficult to precisely determine whether specific microbes are associated with $\mathrm{I} / \mathrm{R}$ development or progression. Here, we for the first time found that the abundance of $L$. murinus was significantly increased in the Res mice compared with the Sen mice, and the fold change was the largest. L. murinus strains have previously been isolated and identified from rat, mice, porcine and canine species, and humans [33,34]. Few reports have indicated the application of $L$. murinus in host health and disease. It has been shown that $L$. murinus may be used as a potential probiotic to reduce the incidence of delayed 
sepsis in neonates [35]. In addition, another study showed that $L$. murinus HU-1 improved the abnormal neural behavior of offspring mice caused by maternal dysregulation [36]. Further, treatment of mice with L. murinus prevented salt-sensitive hypertension by modulating Th17 cells [22]. Furthermore, L. murinus improved colitis by inducing Treg cell differentiation in colitis model [7]. Various L. murinus strains have been further characterized as potential probiotics in the food formulation industry. With increased public interest in $L$. murinus containing probiotics, the impacts of $L$. murinus on intestinal I/R injury are beginning to be unraveled.

At present, macrophages are considered to be a potential therapeutic intervention target for inflammatory diseases and cancer [37]. The switch from M1 to M2 phenotype may reduce intestinal I/R injury [23], and M1 polarization of liver macrophages may be one of the mechanisms of intestinal I/R-induced hepatic injury in mice [38]. However, the mechanisms underlying the specific role of macrophages in intestinal $I / R$ are not fully elucidated. Clodronate-liposome was able to eliminate macrophages and had a certain protective effect on the intestinal morphological damage in the intestinal I/R mouse model, consistent with the results reported in the literature [39]. In addition, L. murinus reduced morphological damage of intestinal organoids injury and LDH levels in the co-culture system of macrophages and small intestinal organoids following $H / R$, but had no obvious effect on these data in the organoids cultured alone, which showed that the protective effect of $L$. murinus on organoid injury depends on the participation of macrophages. 3D intestinal organoid culture was first established in 2009 [40]. Compared with simple intestinal epithelial cell lines, organoids have the physiology of natural intestinal epithelium and functional diversity $[15,41,42]$. Compared with in vivo experiments, organoid systems in vitro avoided the interference of multiple complex factors in vivo and thus are more convenient and accurate. Here, we established an intestinal organoid $\mathrm{H} / \mathrm{R}$ model in vitro to simulate intestinal I/R injury and confirmed that it is reliable for studying the mechanism and treatment of intestinal I/R injury.

In our study, although L. murinus treatment significant decreased in the number of macrophages, compared with I/R in WT mice, L. murinus reduced the proportion of M1 macrophages and increased the M2 polarization of macrophages. In addition, L. murinus significantly increased the proportion of M2 macrophages and TLR2 ${ }^{+}$and IL- $10^{+} \mathrm{M} 2$ macrophages/total M2 macrophages in WT mice following intestinal I/R, but not in TLR2 ${ }^{-/-}$mice. The reported role of TLR2/Myd88 signaling in intestinal I/R injury are controversial. Compared with WT mice, $\mathrm{TLR}^{-/-}$mice have a dysregulated mucosal innate immune response and fail to produce a protective response after intestinal I/R [43]. However, some groups found that TLR2 ${ }^{-/-}$mice have less intestinal damage and inflammation compared with WT mice $[44,45]$. In this study, compared with WT mice, $\mathrm{TLR}^{-/-}$mice fail to produce a protective response after I/R. In addition, Liang et al. revealed that bifidobacteria/ and lactobacilli induced macrophages to secrete IL-10 by activating TLR2 and MyD88 pathways, conferring a protective effect in hosts suffering from inflammation diseases [46]. Another group found that TLR2-mediated secretion of IL-10 and immune suppression in response to phagosome-confined Listeria monocytogenes [47]. Clostridium butyricum directly triggered IL-10 production by intestinal macrophages in inflamed mucosa via the TLR2/MyD88 pathway, thereby preventing experimental colitis in mice [48]. In this study, we found that L. murinus 
alleviates intestinal I/R injury through promoting the release of IL-10 from M2 macrophages via activating TLR2 signaling, which shed light on a novel mechanism of intestinal I/R injury.

Although, differences in the gut microbiota at the individual level may lead to differences in the severity of intestinal I/R injury in patients, the gut microbiome is a complex system where different strains may act together to affect the intestine, and thus there is no clear evidence that one individual strain is the most beneficial. Our pilot study on patients was conducted on a limited number of individuals and needs to be validated in a larger cohort. Moreover, metagenomic and metabolomic analyses and clinical studies need to be performed to confirm the involvement of $L$. murinus in intestinal I/R injury and develop effective probiotics in the future.

\section{Conclusions}

Taken together, our results suggest that gut microbiome is involved in postoperative outcome of intestinal I/R. Furthermore, the present results reveal that $L$. murinus alleviates intestinal I/R injury through promoting the release of IL-10 from M2 macrophages via activating TLR2 signaling, which shed light on a novel mechanism of intestinal I/R injury and suggest that the therapy via targeting microbiome, TLR2 and IL-10 is a promising strategy to prevent intestinal I/R injury.

\section{Abbreviations}

$A B X$, Antibiotic; $A G I$, acute gastrointestinal injury; ANOVA, Analysis of variance; $C P B$, Cardiopulmonary bypass; FMT, Fecal microbiota transplantation; HE, Hematoxylin-eosin; H/R, Hypoxia/reoxygenation; IFABP, Intestinal fatty acid-binding protein; IL-10, Interleukin-10; I/R, Ischemia/reperfusion; LDA, Linear Discriminant Analysis; LDH, Lactate dehydrogenase; LEfSe, Linear discriminant effect size; L. murinus, Lactobacillus murinus; MФ, Macrophages; Myd88, Myeloid differentiation factor 88; Org, Organoid; OTUs, Operational taxonomic units; PCoA, Principal coordinates analysis; PCR, Polymerase chain reaction; SEM, Standard error of mean; Sen, Sensitive; SMA, Superior mesenteric artery; TLR2, Toll-like receptor 2; Res, Resistant; WT, Wild-type.

\section{Declarations}

Ethics approval and consent to participate: The study protocol was approved by the Ethical Committee of Nanfang hospital, Southern Medical University (approval number NFEC-202009-k2-01), and informed consent was obtained from participants.

Consent for publication: Not applicable.

Availability of data and material: The raw sequencing data generated in this study have been deposited in NCBI Sequence Read Archive (http://www.ncbi.nim.nih.gov/sra) under the accession number PRJNA661144. All other data associated with this study are present in the paper or Supplementary Materials. 
Competing interests: All authors declare that they have no competing interests.

Funding: This study was supported by the Key Project of National Natural Science Foundation of China (81730058) and National Natural Science Foundation of China (81671955).

Author contributions: J.J.H, F.D and K.X.L conceived and designed the project. J.J.H, C.L and B.C.Z performed all clinal experiments. F.D and C.L analyzed 16S rRNA gene sequencing data. F.D, X.Y and Z.B.L performed all animal experiments and analyzed all animal data. J.Z, Q.S.S and Z.Z.Y performed histology, PCR, intestinal permeability and ELISA experiments. W.F.L, M.W and S.D.Q collected clinical samples and analyzed data. J.J.H, F.D and K.X.L wrote the paper with the assistance of the other authors.

Acknowledgments: We thank Peng Chen for technical assistance and would like to thank Editage (www.editage.cn) for English language editing.

\section{References}

1. Liara M. Gonzalez AJM, 2 Anthony T. Blikslager1. Animal models of ischemia-reperfusion induced intestinal injury_ progress and promise for translational research. Am J Physiol Gastrointest Liver Physiol. 2015;308(2):G63-75.

2. Rosero $\mathrm{O}$, Onody P, Kovacs T. Impaired intestinal mucosal barrier upon ischemia-reperfusion: "patching holes in the shield with a simple surgical method". 2014;2014(210901.

3. Trompeter M, Brazda T, Remy CT, Vestring T, Reimer P. Non-occlusive mesenteric ischemia: etiology, diagnosis, and interventional therapy. Eur Radiol. 2002;12(5):1179-1187.

4. Pierro A, Eaton S. Intestinal ischemia reperfusion injury and multisystem organ failure. Semin Pediatr Surg. 2004;13(1):11-17.

5. Mowat AM, Agace WW. Regional specialization within the intestinal immune system. Nat Rev Immunol. 2014;14(10):667-685.

6. Nadatani Y, Watanabe T, Shimada S, Otani K, Tanigawa T, Fujiwara Y. Microbiome and intestinal ischemia/reperfusion injury. J Clin Biochem Nutr. 2018;63(1):26-32.

7. Tang C, et al. Inhibition of Dectin-1 Signaling Ameliorates Colitis by Inducing Lactobacillus-Mediated Regulatory T Cell Expansion in the Intestine. Cell host \& microbe. 2015;18(2):183-197.

8. Ren C, et al. Lactic acid bacteria secrete toll like receptor 2 stimulating and macrophage immunomodulating bioactive factors. Journal of Functional Foods. 2020;66(

9. AlQasrawi D, Naser SA. Nicotine Modulates MyD88-Dependent Signaling Pathway in Macrophages during Mycobacterial Infection. Microorganisms. 2020;8(11):

10. Dong J, et al. Oral treatment with SEW2871, a sphingosine-1-phosphate type 1 receptor agonist, ameliorates experimental colitis in interleukin-10 gene deficient mice. Clin Exp Immunol. 2014;177(1):94-101. 
11. Yang Z, et al. Knockdown of TNFalpha alleviates acute lung injury in rats with intestinal ischemia and reperfusion injury by upregulating IL10 expression. Int J Mol Med. 2018;42(2):926-934.

12. Ellett JD, et al. Murine Kupffer cells are protective in total hepatic ischemia/reperfusion injury with bowel congestion through IL-10. J Immunol. 2010;184(10):5849-5858.

13. Zhou J, et al. Intestinal ischemia/reperfusion enhances microglial activation and induces cerebral injury and memory dysfunction in rats. Crit Care Med. 2012;40(8):2438-2448.

14. Zwarycz B, et al. IL22 Inhibits Epithelial Stem Cell Expansion in an Ileal Organoid Model. Cellular and molecular gastroenterology and hepatology. 2019;7(1):1-17.

15. Deng F, et al. Interleukin-10 expands transit-amplifying cells while depleting Lgr5(+) stem cells via inhibition of Wnt and notch signaling. Biochemical and biophysical research communications. 2020;

16. Reintam Blaser A, et al. Gastrointestinal function in intensive care patients: terminology, definitions and management. Recommendations of the ESICM Working Group on Abdominal Problems. Intensive Care Med. 2012;38(3):384-394.

17. Chen $P$, et al. Supplementation of saturated long-chain fatty acids maintains intestinal eubiosis and reduces ethanol-induced liver injury in mice. Gastroenterology. 2015;148(1):203-214.e216.

18. Hu J, et al. Enteric dysbiosis-linked gut barrier disruption triggers early renal injury induced by chronic high salt feeding in mice. Exp Mol Med. 2017;49(8):e370.

19. Caporaso JG, et al. QIIME allows analysis of high-throughput community sequencing data. Nat Methods. 2010;7(5):335-336.

20. Segata N, et al. Metagenomic biomarker discovery and explanation. Genome Biol. 2011;12(6):R60.

21. Asshauer KP, Wemheuer B, Daniel R, Meinicke P. Tax4Fun: predicting functional profiles from metagenomic 16S rRNA data. Bioinformatics. 2015;31(17):2882-2884.

22. Wilck N, et al. Salt-responsive gut commensal modulates TH17 axis and disease. Nature. 2017;551(7682):585-589.

23. Liu WF, et al. Treatment with Recombinant Trichinella spiralis Cathepsin B-like Protein Ameliorates Intestinal Ischemia/Reperfusion Injury in Mice by Promoting a Switch from M1 to M2 Macrophages. J Immunol. 2015;195(1):317-328.

24. Aydin S, Caylan R, Aydin K, Yulug E, Yenilmez E, Koksal I. The influence of G-CSF addition to antibiotic treatment of experimental sepsis on pulmonary tissue. $\mathrm{J}$ Natl Med Assoc. 2005;97(11):1489-1495.

25. Gu J, et al. Dexmedetomidine provides renoprotection against ischemia-reperfusion injury in mice. Crit Care. 2011;15(3):R153.

26. Shen XD, et al. Toll-like receptor and heme oxygenase-1 signaling in hepatic ischemia/reperfusion injury. Am J Transplant. 2005;5(8):1793-1800.

27. Adamik B, Kubler A, Gozdzik A, Gozdzik W. Prolonged Cardiopulmonary Bypass is a Risk Factor for Intestinal Ischaemic Damage and Endotoxaemia. Heart, lung \& circulation. 2017;26(7):717-723. 
28. Struck R, Wittmann M, Müller S, Meybohm P, Müller A, Bagci S. Effect of Remote Ischemic Preconditioning on Intestinal Ischemia-Reperfusion Injury in Adults Undergoing On-Pump CABG Surgery: A Randomized Controlled Pilot Trial. Journal of Cardiothoracic and Vascular Anesthesia. 2018;32(3):1243-1247.

29. Blaser A, Padar M, Tang J, Dutton J, Forbes A. Citrulline and intestinal fatty acid-binding protein as biomarkers for gastrointestinal dysfunction in the critically ill. Anaesthesiology intensive therapy. 2019;51(3):230-239.

30. Zou L, et al. Intestinal fatty acid-binding protein as a predictor of prognosis in postoperative cardiac surgery patients. Medicine. 2018;97(33):e11782.

31. Wang F, et al. Temporal variations of the ileal microbiota in intestinal ischemia and reperfusion. Shock. 2013;39(1):96-103.

32. Yoshiya $\mathrm{K}$, et al. Depletion of gut commensal bacteria attenuates intestinal ischemia/reperfusion injury. Am J Physiol Gastrointest Liver Physiol. 2011;301(6):G1020-1030.

33. Perelmuter K, Fraga M, Zunino P. In vitro activity of potential probiotic Lactobacillus murinus isolated from the dog. Journal of applied microbiology. 2008;104(6):1718-1725.

34. Rossi M, Martínez-Martínez D, Amaretti A, Ulrici A, Raimondi S, Moya A. Mining metagenomic whole genome sequences revealed subdominant but constantLactobacilluspopulation in the human gut microbiota. Environmental Microbiology Reports. 2016;8(3):399-406.

35. Singer JR, et al. Preventing dysbiosis of the neonatal mouse intestinal microbiome protects against late-onset sepsis. Nature medicine. 2019;25(11):1772-1782.

36. Lebovitz $\mathrm{Y}$, et al. Lactobacillus rescues postnatal neurobehavioral and microglial dysfunction in a model of maternal microbiome dysbiosis. Brain, behavior, and immunity. 2019;81(617-629.

37. Ponzoni M, Pastorino F, Di Paolo D, Perri P, Brignole C. Targeting Macrophages as a Potential Therapeutic Intervention: Impact on Inflammatory Diseases and Cancer. International journal of molecular sciences. 2018;19(7):

38. Wen S, et al. HMGB1-associated necroptosis and Kupffer cells M1 polarization underlies remote liver injury induced by intestinal ischemia/reperfusion in rats. FASEB journal : official publication of the Federation of American Societies for Experimental Biology. 2020;34(3):4384-4402.

39. Chen Y, Lui VC, Rooijen NV, Tam PK. Depletion of intestinal resident macrophages prevents ischaemia reperfusion injury in gut. Gut. 2004;53(12):1772-1780.

40. Sato T, et al. Single Lgr5 stem cells build crypt-villus structures in vitro without a mesenchymal niche. Nature. 2009;459(7244):262-265.

41. Pleguezuelos-Manzano C, et al. Mutational signature in colorectal cancer caused by genotoxic pks(+) E. coli. Nature. 2020;580(7802):269-273.

42. Nanki K, et al. Somatic inflammatory gene mutations in human ulcerative colitis epithelium. Nature. 2020;577(7789):254-259. 
43. Aprahamian CJ, Lorenz RG, Harmon CM, Dimmit RA. Toll-like receptor 2 is protective of ischemiareperfusion-mediated small-bowel injury in a murine model. Pediatr Crit Care Med. 2008;9(1):105109.

44. Goering J, Pope MR, Fleming SD. TLR2 Regulates Complement-Mediated Inflammation Induced by Blood Loss During Hemorrhage. Shock. 2016;45(1):33-39.

45. Pope MR, Fleming SD. TLR2 modulates antibodies required for intestinal ischemia/reperfusioninduced damage and inflammation. J Immunol. 2015;194(3):1190-1198.

46. Liang $\mathrm{H}$, et al. Lactobacilli and bifidobacteria derived from infant intestines may activate macrophages and lead to different IL-10 secretion. Biosci Biotechnol Biochem. 2020;84(12):25582568.

47. Nguyen BN, Chavez-Arroyo A, Cheng MI, Krasilnikov M, Louie A, Portnoy DA. TLR2 and endosomal TLR-mediated secretion of IL-10 and immune suppression in response to phagosome-confined Listeria monocytogenes. PLoS Pathog. 2020;16(7):e1008622.

48. Hayashi A, et al. A single strain of Clostridium butyricum induces intestinal IL-10-producing macrophages to suppress acute experimental colitis in mice. Cell host \& microbe. 2013;13(6):711722.

\section{Figures}



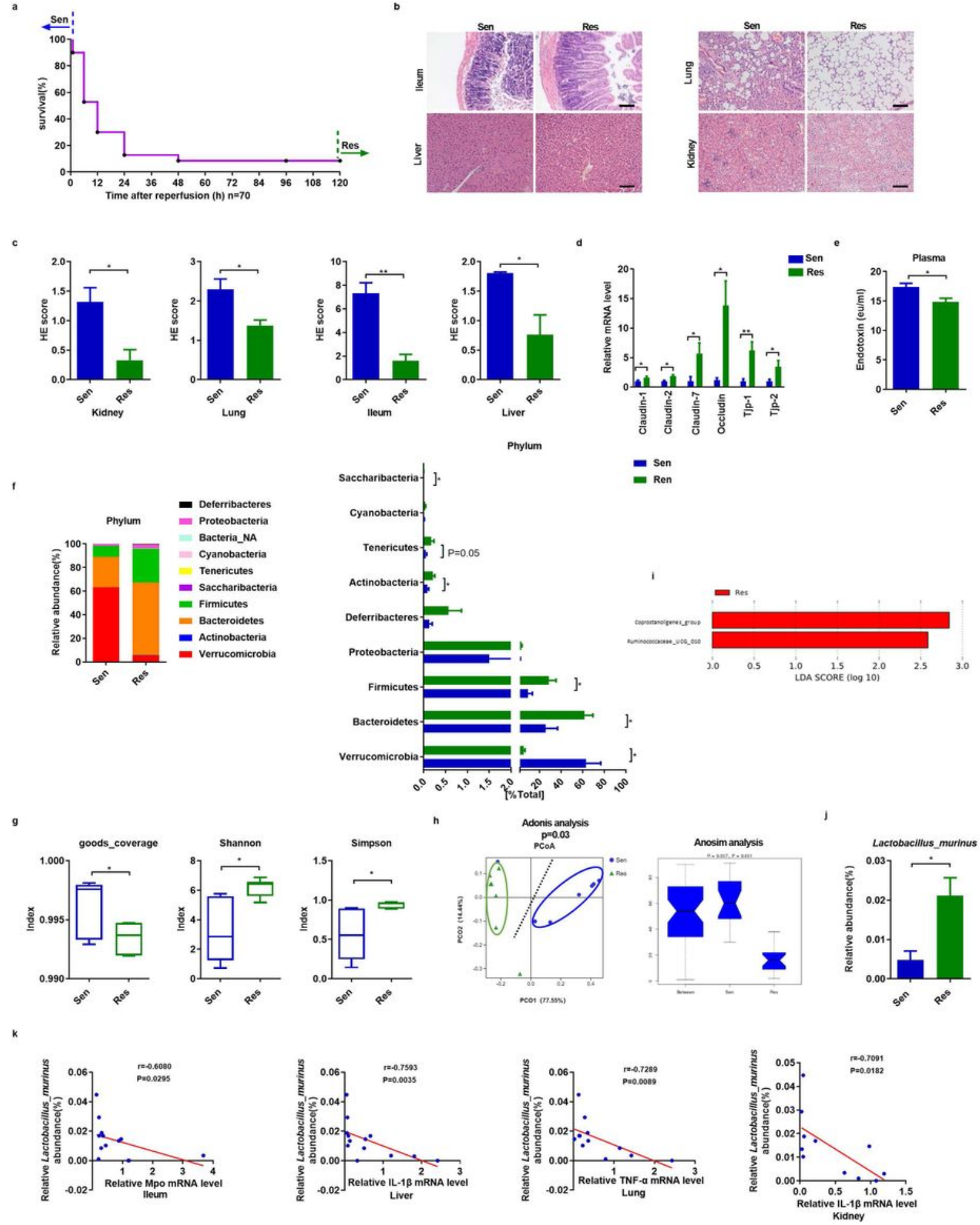

\section{Figure 1}

Characterization of tissue injury and the gut microbiota in Sen and Res mice. 6-8-weeks male C57BL/6 mice underwent intestinal ischemia for $60 \mathrm{~min}$ and the survival rate was observed after reperfusion. We defined mice that died within one hour after reperfusion as Sen mice, and those that survived for 5 days as Res mice. a Changes in survival rate $(n=70)$ have been shown. b, c HE staining of the ileum, liver, lung and kidney to visualize the histomorphological features and quantification analysis. Scale bar=100 $\mu \mathrm{m}$. $d$ 
The mRNA levels of tight junction proteins in the ileum. e Relative plasma endotoxin level. $f$ Relative bacterial abundance at the phyla level in the feces of mice. $g$ Alpha diversity indices. h PCoA based on the weighted UniFrac analysis of operational taxonomic units (OTUs)and anosim analysis. i Histogram of the LDA score showing the enriched bacteria in the gut microbiome of the Res mice. $j$ The relative abundance of $L$. murinus at the species level in the Sen and Res mice. $k$ The correlation between the mRNA levels of inflammatory factors in the ileum, liver, lung and kidney and the abundance of L. murinus in the Sen and Res mice. The results are expressed as the mean \pm SEM. $n=7 .{ }^{\star \star \star} p<0.001,{ }^{\star \star} p<0.01$, ${ }^{*} p<0.05$ were determined by two-tailed Student's $t$ test in (c-h), adonis analysis and anosim analysis in (i), spearman analysis in (k). LDA, Linear Discriminant Analysis; LDA, Linear Discriminant Analysis; L. murinus, Lactobacillus murinus; OTUs, operational taxonomic units; PCOA, Principal Coordinates Analysis; Sen, sensitive; Res, resistant. 

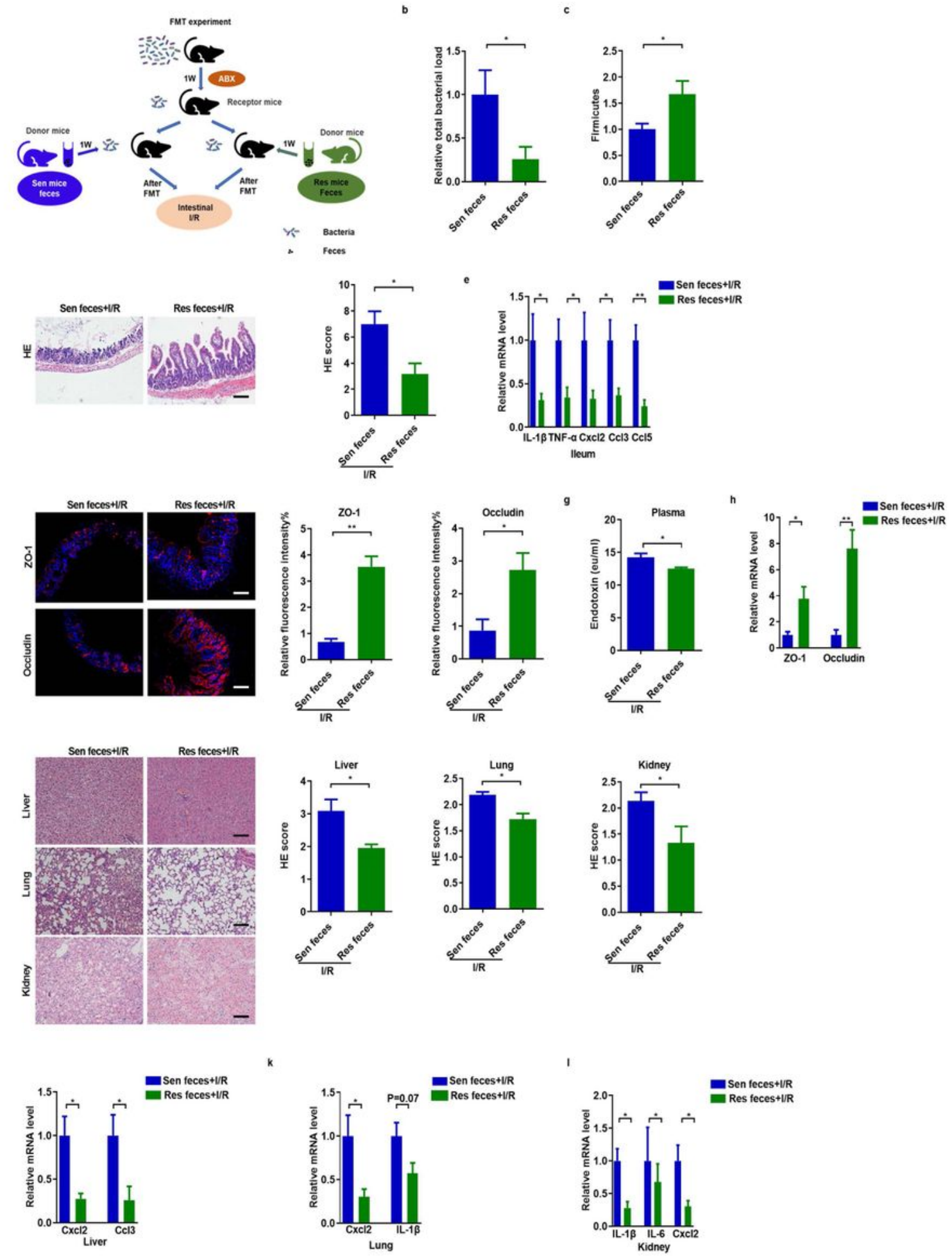

\section{Figure 2}

Gut microbiota from Res mice independently alleviates I/R-induced intestinal, liver, lung, and kidney tissue damage. a FMT experimental design. Mice were subjected to intestinal ischemia for $60 \mathrm{~min}$ and reperfusion for 120 min after 1 week of transplantation. b, c The total bacterial load and the abundance of Firmicutes in the feces 1 week after transplantation. $d$ HE staining in the ileum. Scale bar $=100 \mu \mathrm{m}$. $\mathrm{e}$ The mRNA levels of proinflammatory factors in the ileum. f-h Tight junction mRNA levels and protein 
levels in the ileum and relative plasma endotoxin level. Scale bar=100 $\mu \mathrm{m}$. i HE staining in the liver, lung and kidney and the quantification analysis. Scale bar $=100 \mu \mathrm{m}$. $j-1$ The mRNA levels of proinflammatory factors in the liver, lung and kidney. The results are expressed as the mean \pm SEM. $n=8 .{ }^{* \star *} p<0.001$, ${ }^{*} \mathrm{p}<0.01,{ }^{*} \mathrm{p}<0.05$ were determined using two-tailed Student's $\mathrm{t}$ test. FMT, fecal microbiota transplantation; HE, Hematoxylin-eosin; I/R, ischemia/reperfusion.
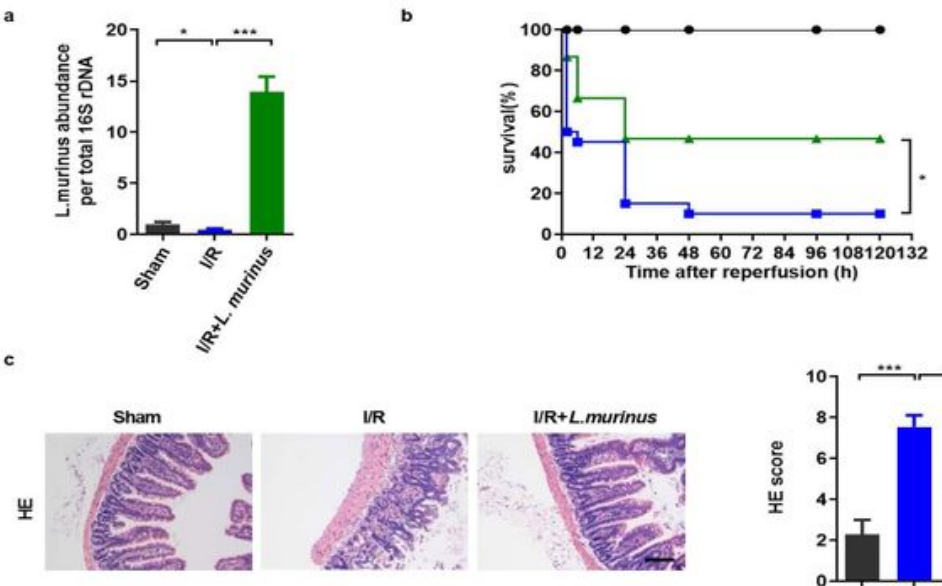

$\rightarrow$ sham

$-1 / R$
- $/ R+$ L. murinus
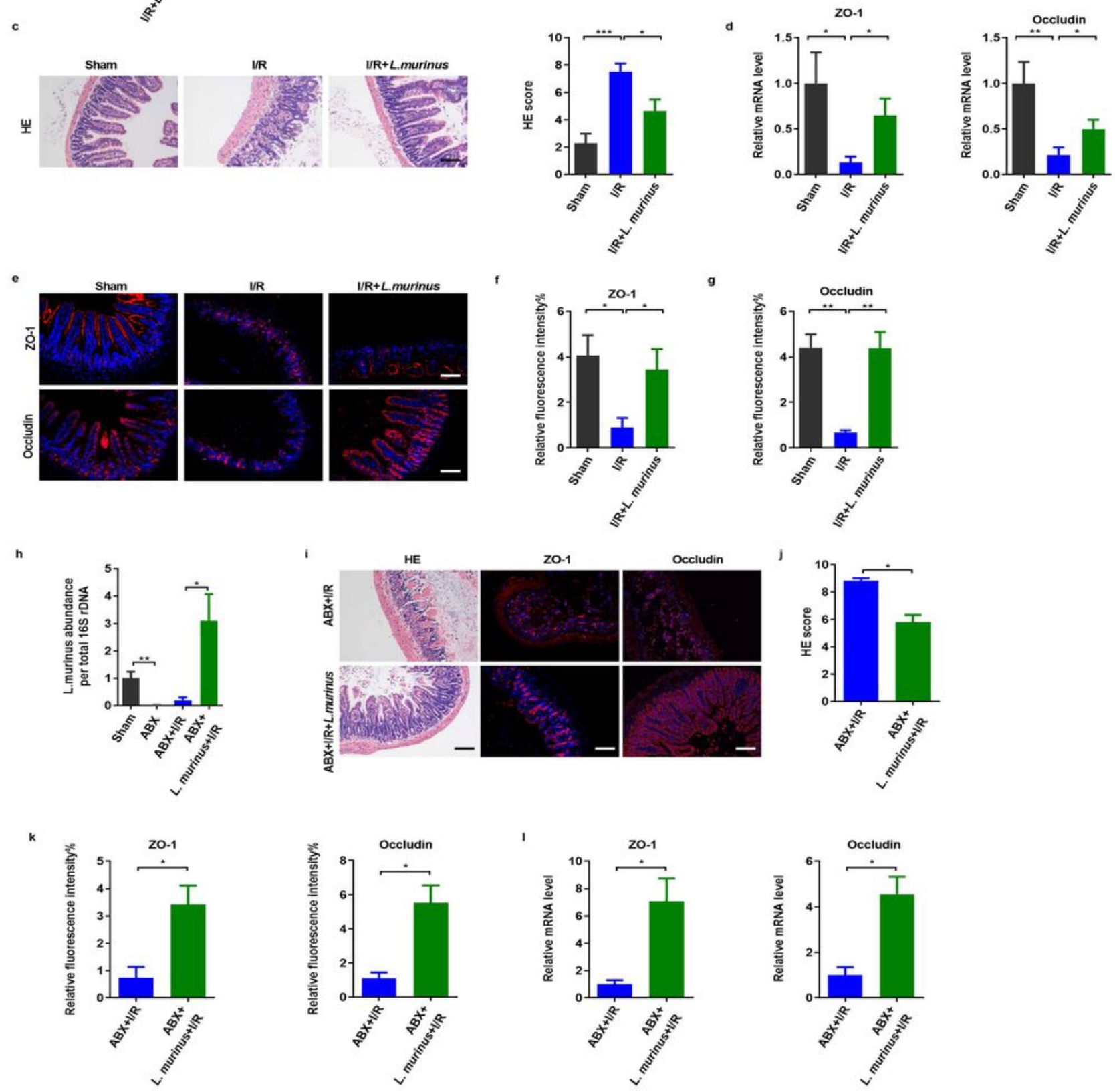

Figure 3 
L. murinus improves intestinal I/R-induced intestinal injury. a Relative abundance of L. murinus in the cecum by real-time PCR. $b$ Effect of $L$. murinus on survival rate. Mice were subjected to ischemia of SMA for 60 min, and survival was monitored on day 5 after reperfusion $(n=15)$. c HE staining in the ileum. Representative quantification on the right. Scale bar $=100 \mu \mathrm{m}$. d-g The relative mRNA and protein levels of ZO-1 and Occludin in the ileum were measured by immunofluorescent staining. Scale bar $=100 \mu \mathrm{m} . \mathrm{h}$ Relative abundance of L. murinus in the cecum after ABX clearance experiment. i-k HE staining and ZO-1 and Occludin immunofluorescent staining in the ileum. Representative quantification on the right. Scale bar $=100 \mu \mathrm{m}$. I Tight junction mRNA levels in the ileum. The results are expressed as the mean \pm SEM. $\mathrm{n}=$ 8. ${ }^{* \star *} p<0.001,{ }^{* \star} p<0.01,{ }^{*} p<0.05$ were determined by one-way ANOVA (Tukey's test) and Log-Rank test in (b). ABX, Antibiotic; ANOVA, Analysis of variance; I/R, ischemia/reperfusion; L. murinus, Lactobacillus murinus; SMA, Superior mesenteric artery. 

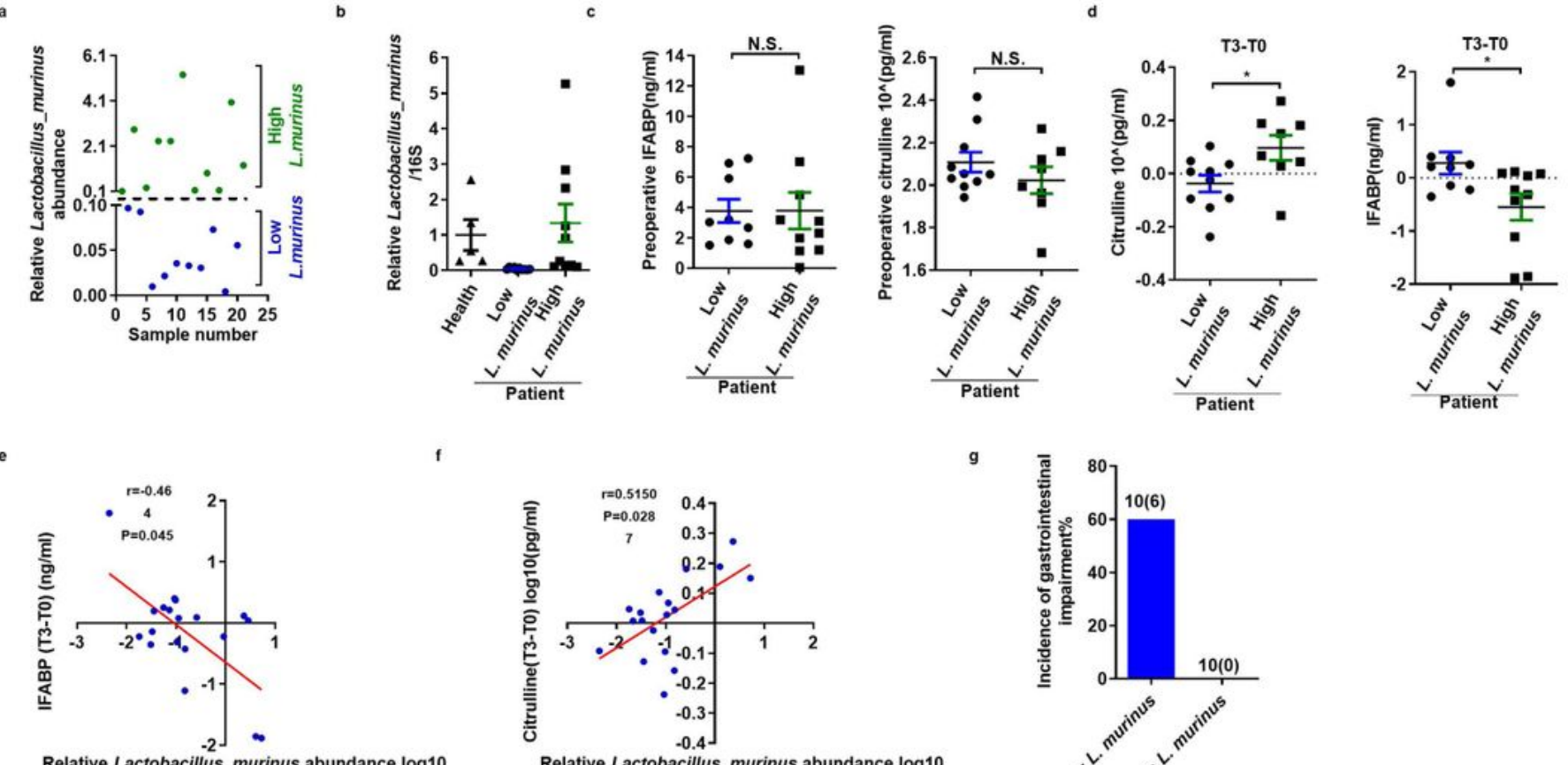

Relative Lactobacillus_murinus abundance $\log 10$

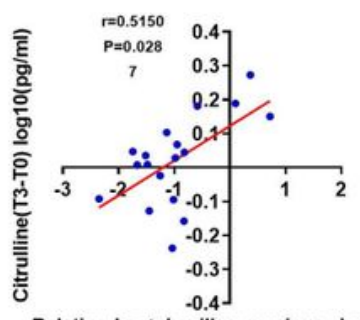

g
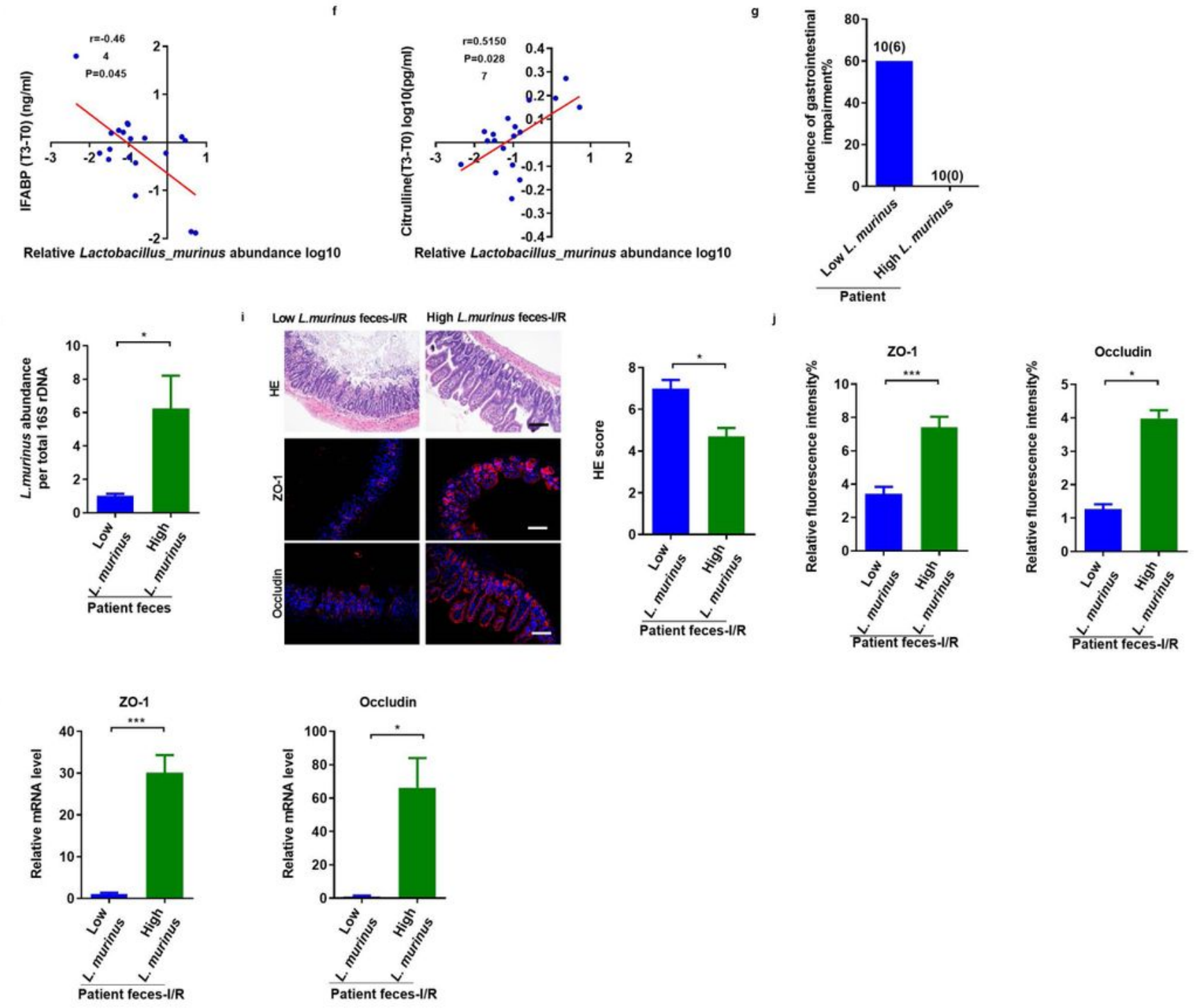

\section{Figure 4}

Relationship between L. murinus and postoperative intestinal injury in patients undergoing cardiopulmonary bypass surgery. $a, b$ Relative abundance of $L$. murinus in preoperative feces of CPB patients $(n=20)$ and health individuals $(n=6)$. c the level of IFABP and citrulline in serum before surgery between low $L$. murinus abundance group and high $L$. murinus abundance group $(n=10)$. $d$ Changes in IFABP and citrulline concentrations at 6 hours postoperatively $(n=10)$. e, $f$ Correlation of relative L. 
murinus abundance with serum IFABP level and citrulline level. $g$ Occurrence of gastrointestinal injury in patients undergoing CPB. $h$ Relative abundances of $L$. murinus in the feces by real-time PCR between low L. murinus feces group and high L. murinus feces group. $(n=8)$ i, j HE staining and ZO-1 and Occludin immunofluorescent staining in the ileum and representative quantification. Scale bar $=100 \mu \mathrm{m}(\mathrm{n}=8) \mathrm{k}$ Relative mRNA levels of ZO-1 and Occludin in the ileum. The results are expressed as the mean \pm SEM. ${ }^{\star * \star} p<0.001,{ }^{* \star} p<0.01,{ }^{*} p<0.05$ were determined by two-tailed Student's $t$ test and spearman analysis in (ef). CPB, cardiopulmonary bypass; IFABP, Intestinal fatty acid-binding protein; L. murinus, Lactobacillus murinus.
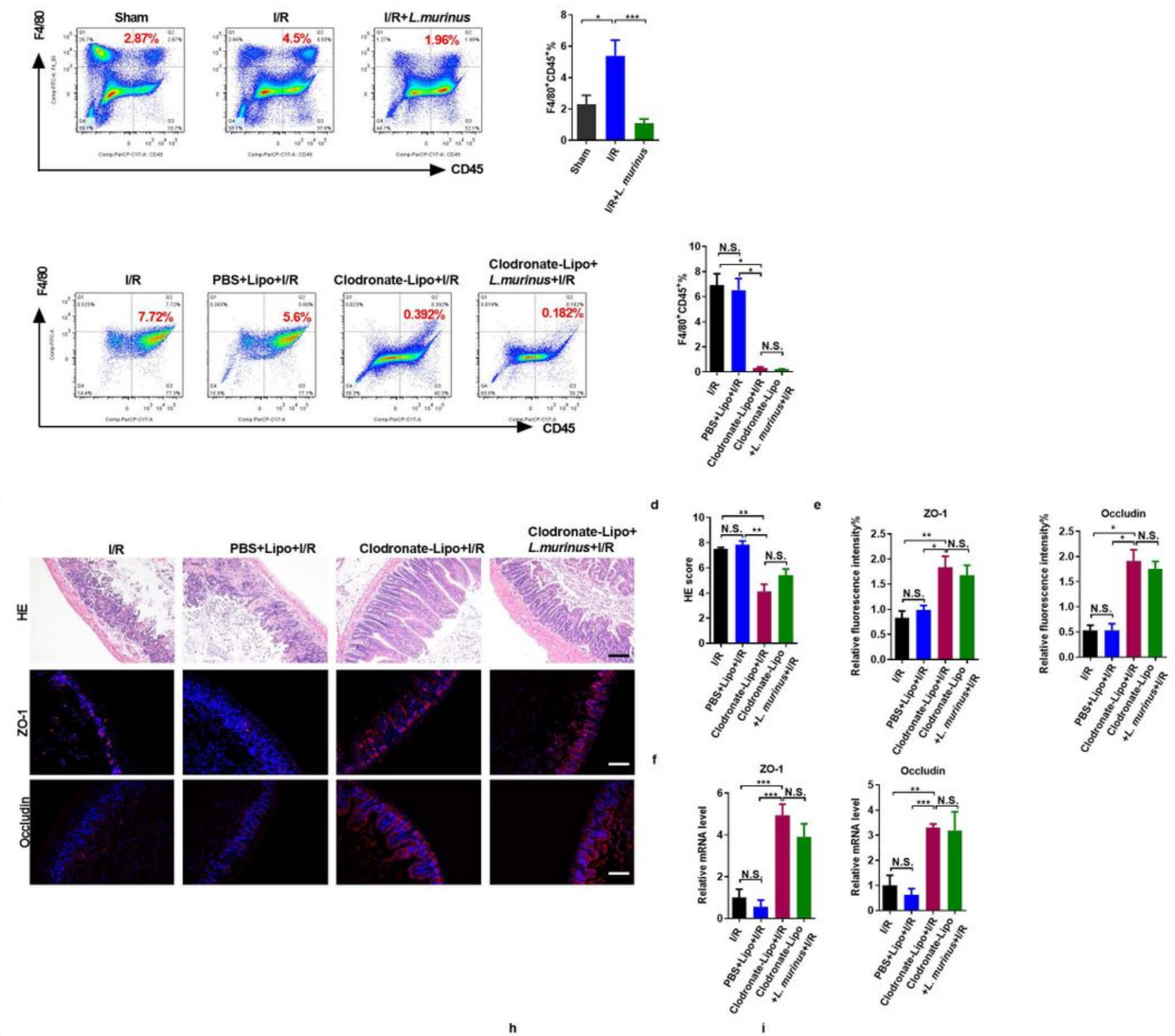

g
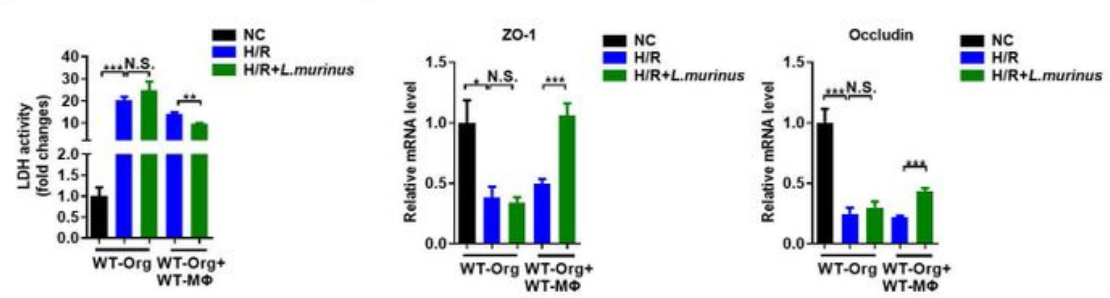

Figure 5 
Improvement in intestinal I/R injury by L. murinus depends on the participation of macrophages. a Peritoneal macrophages were analyzed by flow cytometry for F4/80+CD45+ cells in mice in the sham group, I/R group or IR+L. murinus group $(n=3-4)$. Representative quantification on the right. b Peritoneal macrophage was analyzed by flow cytometry for F4/80+CD45+ cells in mice ( $n=3-4)$. c-f HE staining and ZO-1 and Occludin immunofluorescent staining in the ileum and representative quantification. Scale bar $=100 \mu \mathrm{m}$. g-i HE staining, relative LDH levels and tight junction mRNA levels were detected in organoids cultured alone and co-culture with macrophages after $\mathrm{H} / \mathrm{R}$. Scale bar $=20 \mu \mathrm{m}$. The results are expressed as the mean \pm SEM. $n=6$. ${ }^{* *} p<0.001,{ }^{* \star} p<0.01,{ }^{*} p<0.05$ were determined by one-way ANOVA (Tukey's test) and Log-Rank test in (b). ANOVA, Analysis of variance; HE, Hematoxylin-eosin; H/R, hypoxia/reoxygenation; I/R, ischemia/reperfusion; LDH, Lactate dehydrogenase; L. murinus, Lactobacillus murinus. 

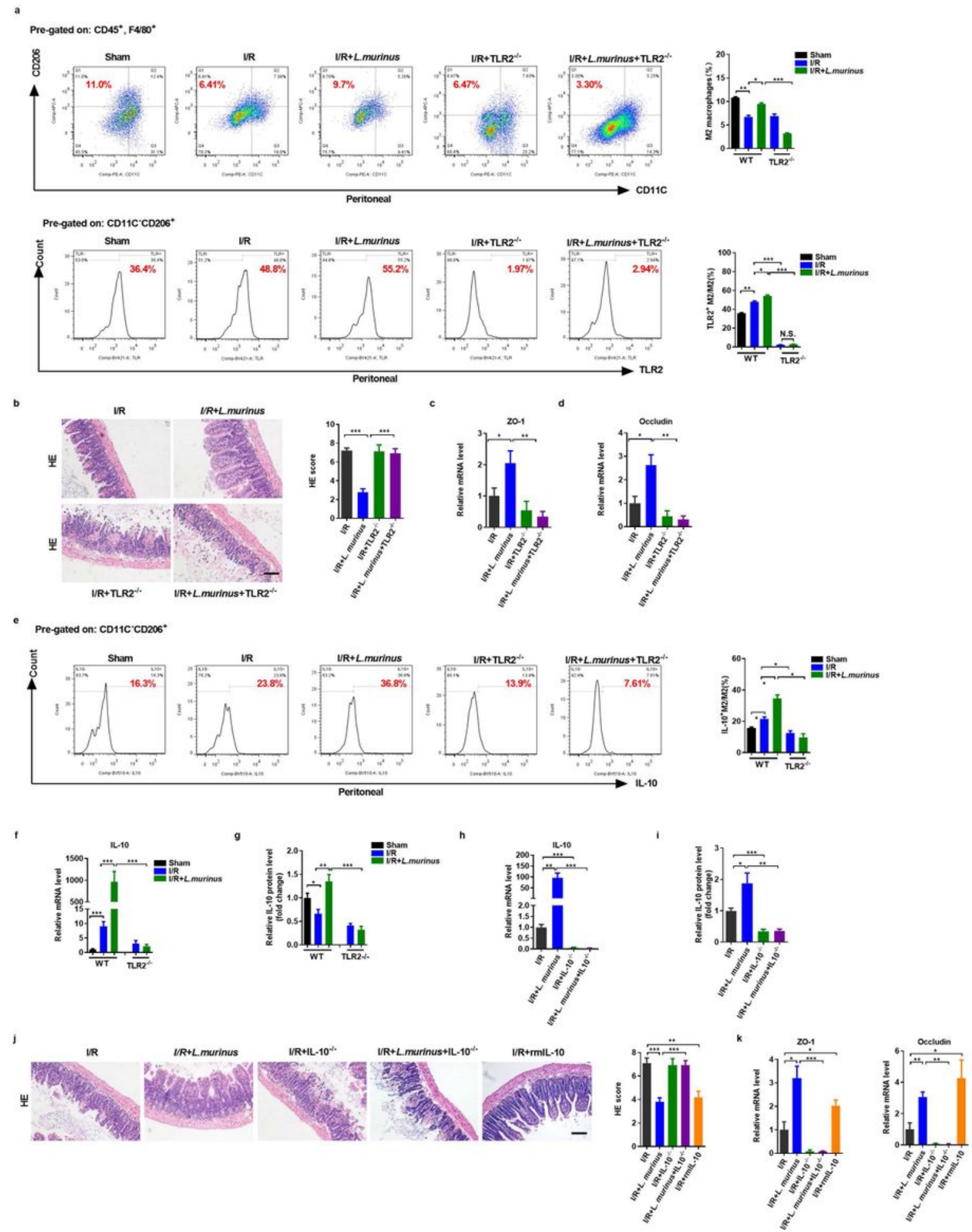

Figure 6

L. murinus promotes the release of IL-10 from M2 macrophages through TLR2 to alleviate intestinal I/R injury. a Peritoneal macrophages were analyzed by flow cytometry for F4/80+CD206+CD11C- cells and the expression of TLR2 on the surface M2 macrophages in mice in the sham group, I/R group, IR+L. murinus group, I/R+TLR2-/- group and I/R+L. murinus+TLR2-/- group. Representative quantification on the right $(n=3-4)$. b HE staining in the ileum. Scale bar=100 $\mu \mathrm{m}$. c, $d$ The relative mRNA levels of ZO-1 
and Occludin in the ileum. e The proportion of IL-10+ M2 macrophages/total M2 macrophages were analyzed by flow cytometry in mice with sham group, I/ R group, IR+L. murinus group, I/R+TLR2-/- group and I/R+L. murinus+TLR2-/- group. Representative quantification on the right $(n=3-4)$. $f-i$ The relative mRNA and protein level of IL-10 in the ileum. j HE staining in the ileum and the quantification analysis. Scale bar $=100 \mu \mathrm{m}$. $\mathrm{k}$ The relative mRNA levels of ZO-1 and Occludin in the ileum. The results are expressed as the mean \pm SEM. $n=8 * \star \star p<0.001,{ }^{* \star} p<0.01,{ }^{*} p<0.05$ were determined by one-way ANOVA (Tukey's test). ANOVA, Analysis of variance; HE, Hematoxylin-eosin; IL-10, Interleukin-10; I/R, ischemia/reperfusion. L. murinus, Lactobacillus murinus. TLR2, Toll-like receptor 2. 


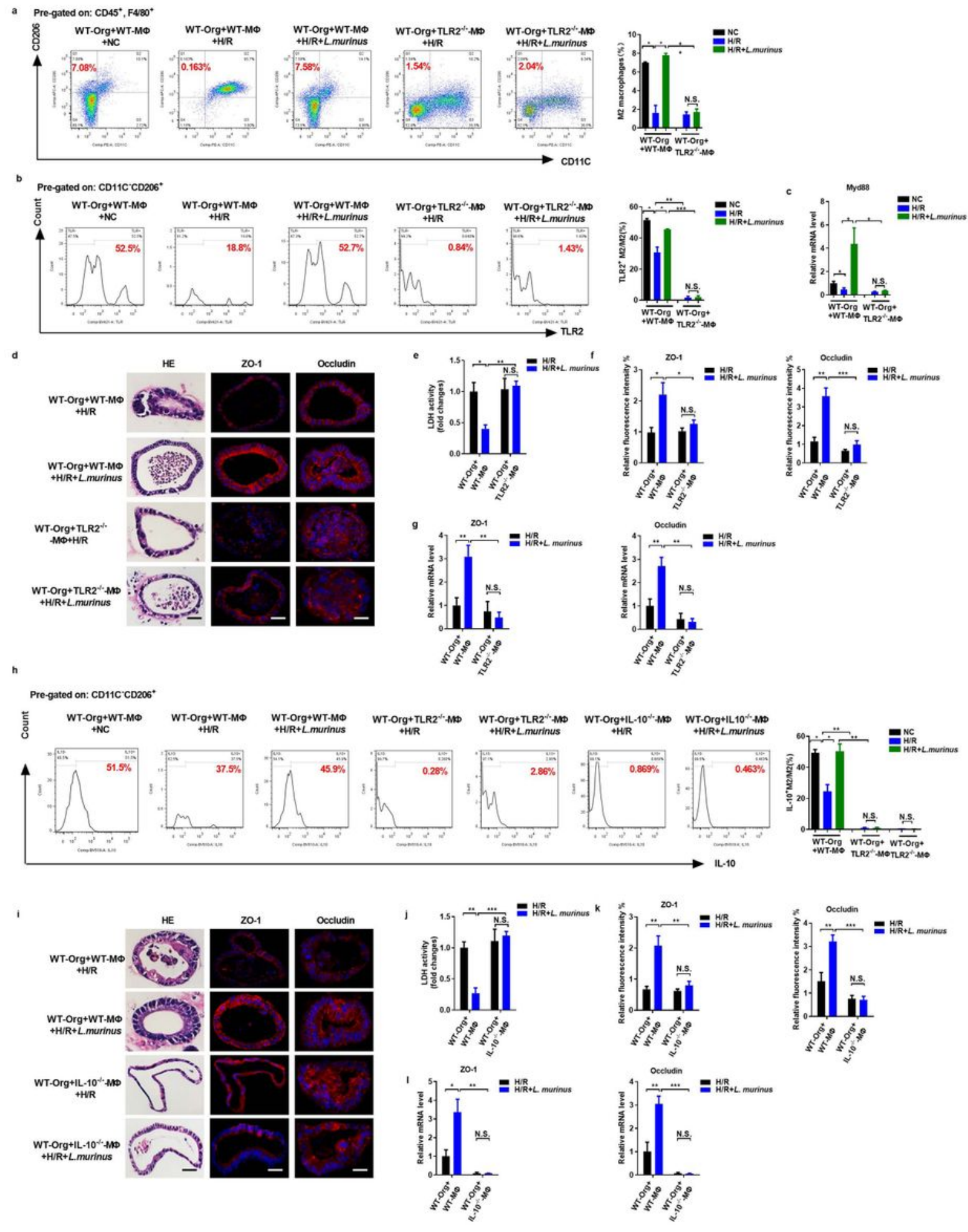

Figure 7

L. murinus promotes the release of IL-10 by M2 macrophages through TLR2 to reduce organoid H/R injury. $a, b$ Macrophages were analyzed by flow cytometry for F4/80+CD206+CD11C- cells and the expression of TLR2 on the surface M2 macrophages in the co-culture system of WT-Org and WT-MФ or WT-Org and TLR2-/-MФ. Representative quantification on the right $(n=3-4)$. c The relative mRNA level of Myd88 in macrophages. d HE and ZO-1 and Occludin immunofluorescent staining in the organoids. Scale 
bar $=20 \mu \mathrm{m}$. e The relative LDH levels were detected in organoids. $\mathrm{f}, \mathrm{g}$ The relative ZO-1 and Occludin fluorescence intensity and mRNA levels of ZO-1 and Occludin in the organoids. $\mathrm{h}$ The proportion of IL$10+\mathrm{M} 2$ macrophages/total M2 macrophages were analyzed by flow cytometry in the co-culture system of WT-Org and WT-MФ or WT-Org and TLR2-/-MФ or WT-Org and IL-10-/-MФ. Representative quantification on the right $(n=3-4)$. i HE and ZO-1 and Occludin immunofluorescent staining in the organoids. Scale bar $=20 \mu \mathrm{m}$. $j$ The relative LDH levels were detected in organoids. $k$, I The relative ZO- 1 and Occludin fluorescence intensity and mRNA levels of ZO-1 and Occludin in the organoids. The results are expressed as the mean \pm SEM. $n=6 .{ }^{* \star} p<0.001,{ }^{* \star} p<0.01,{ }^{*} p<0.05$ were determined by one-way ANOVA (Tukey's test). ANOVA, Analysis of variance; H/R, hypoxia/reoxygenation; IL-10, Interleukin-10; LDH, Lactate dehydrogenase; L. murinus, Lactobacillus murinus; Myd88, myeloid differentiation factor 88; TLR2, Tolllike receptor 2. WT-Org, organoid extracted from WT mice; WT-MФ, macrophages extracted from WT mice; TLR2-/-MФ, macrophages extracted from TLR2-/- mice; IL-10-/--MФ, macrophages extracted from IL10-/mice.

\section{Supplementary Files}

This is a list of supplementary files associated with this preprint. Click to download.

- Additionalfile1.pdf

- Additionalfile2.pdf

- Additionalfile3.pdf

- Additionalfile4.pdf 